Communications in Physics, Vol. 28, No. 2 (2018), pp. 169-177

DOI:10.15625/0868-3166/28/2/11800

\title{
OPTIMAL CARRIER CONCENTRATION FOR HIGH THERMOELECTRIC PERFORMANCE OF LEAD SUBSTITUTED BISMUTH TELLURIDE IN P-TYPE DOPING
}

\author{
TRAN VAN QUANG ${ }^{\dagger}$
}

Department of Physics, University of Transport and Communications, Hanoi, Vietnam

${ }^{\dagger} E$-mail: tkuangv@gmail.com

Received 15 March 2018

Accepted for publication 8 May 2018

Published 16 June 2018

\begin{abstract}
Bi}_{2} \mathrm{Te}_{3}$ and its alloys are the well-known state-of-the-art thermoelectric materials operating at around room temperature. With lead substituted, the newly formed quasi-binary compound $\mathrm{PbBi}_{4} \mathrm{Te}_{7}$, shows relatively high electrical conductivity and Seebeck coefficient. In this report, we employed the solution of the Boltzmann Transport Equation in a constant relaxation-time approximation within a first-principles density-functional-theory calculation to explore the role of the electronic thermal conductivity, $\kappa_{e}$, on the thermoelectric performance of the compound with p-type doping. Results show that $\kappa_{e}$ increases drastically with the increases of both temperature and carrier concentration. Even the power factor has been found to be markedly improved with the increase of the carrier concentration, a rapid increase of $\kappa_{e}$ emerges as a big hindrance to improve the dimensionless figure of merit, ZT, of the compound. This is responsible for the limit of ZT. The larger ZT is found in low temperatures and carrier concentrations. The highest ZT of about 0.48 occurs at $223 \mathrm{~K}$ and at the carrier concentration of $6 \times 10^{17} \mathrm{~cm}^{-3}$. At room temperature the maximum ZT is slightly smaller. We demonstrated that at a particular temperature to maximize the thermoelectric performance of the compound, the carrier concentration must be optimized. Results show that the compound with p-type doping is a promising thermoelectric materials operating at around room temperature.
\end{abstract}

Keywords: first-principles calculation, thermoelectric materials, p-type doping $\mathrm{PbBi}_{4} \mathrm{Te}_{7}$, electronic thermal conductivity.

Classification numbers: 31.15.A-; 71.15.Mb; 72.20.Pa; 84.60.Rb; 71.20.-b.

(C)2018 Vietnam Academy of Science and Technology 


\section{INTRODUCTION}

The future now is dealing with the energy crisis and environmental impacts of fossil fuels. The renewable energy resources are therefore demanded. One of the methods is to convert unused waste heat directly into electricity by utilizing the thermoelectric (TE) effect [1]. Thermoelectric modules have been known to operate friendly with environment and to easily hybrid with electronic devices. In spite of that, the practical applications have still been inefficient due to the poor performance. The thermoelectric performance of a material or a device is determined by the dimensionless figure of merit, which is $[2,3]$

$$
Z T=\frac{\sigma S^{2} T}{\kappa}
$$

where $S$ is the Seebeck coefficient, $T$ the temperature, $\sigma$ the electrical conductivity and $\kappa=\kappa_{e}+\kappa_{L}$ is the total thermal conductivity which includes the electronic thermal conductivity $\kappa_{e}$ and the lattice thermal conductivity $\kappa_{L}$. To improve $Z T, S$ and $\sigma$ are demanded to be large whereas $\kappa$ must be low. Nonetheless, these coefficients have interrelationship. The increase of $\sigma$ usually reduces $S$ and increases $K$ and vice versa. Thus, the highest $Z T$ values of typical thermoelectric materials operating at room temperature found so far are only around unity or even lower [4]. Many methods have been introduced to solve the problem including making supperlattices [5], defects, element substitutions [6], alloy compositions, strains, tuning band structures, etc. [4,6-8]. The best TE materials operating at around room temperature have been found in chalcogenide compounds. The element substitutions in these compounds have been found to manifest many potential effects which arise from the change of band near Fermi level and the increase of phonon scattering [9-12].

Recently, the class of compounds $\mathrm{A}^{I V} \mathrm{Te}-\mathrm{Bi}_{2} \mathrm{Te}_{3}\left(\mathrm{~A}^{I V}=\mathrm{Pb}, \mathrm{Ge}, \mathrm{Sn}\right)$ was reported to have a complex crystalline structure which significantly enhances phonon scattering at long-period leading to the low lattice thermal conductivities. In addition, the high density of states near the Fermi energy gives rise to a rather large Seebeck coefficient $[13,14] . \mathrm{PbBi}_{4} \mathrm{Te}_{7}$ in the class reveals an impressive high power factor. Therewith, its lattice thermal conductivity is relative small $[12,13,15-17]$. Recently, it has been demonstrated that the power factor of the compound can be significantly improved by increasing the carrier concentration and temperature [13]. Nonetheless, the discussion on the effect of the thermal conductivity was neglected [13]. In that scene, the suggestion was raised by the general optimized carrier concentration to maximize the TE performance, technically relying on the power factor $S^{2} \sigma$, not on the figure of merit, ZT. Since the thermal conductivity was unknown. The effect of thermal conductivity on the TE performance of the compound therefore is still under investigation. The important fact stems from its electronic component, $\kappa_{e}$. As reported, the increasing power factor originates also from the electrical conductivity [13]. According to the Wiedemann-Franz law, this leads to a rapid increase of $\kappa_{e}$ which is detrimental for ZT. The question emerged is what level of this effect on the TE performance of $\mathrm{PbBi}_{4} \mathrm{Te}_{7}$. In this work, we present our calculation of $\kappa_{e}$ as a function of temperature $T$ and carrier concentration $n$ by utilizing the semi-classical Boltzmann Transport Equation. We demonstrate that the electronic thermal conductivity plays an important role to determine the TE performance of the compound. Accordingly, a big ZT is found in low carrier concentrations due to the small

$\kappa_{e}$. We show the optimal carrier concentrations for various temperatures and figure out the best operating temperature for the TE performance of the compound. 


\section{CRYSTAL STRUCTURE AND COMPUTATIONAL METHOD}

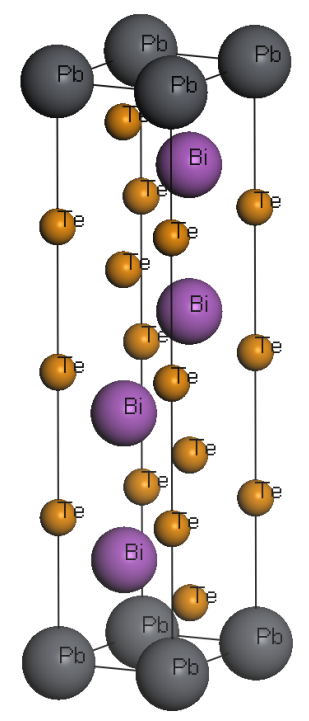

Fig. 1. Primitive cell of $\mathrm{PbBi}_{4} \mathrm{Te}_{7}$ crystal structure.

$\mathrm{PbBi}_{4} \mathrm{Te}_{7}$ compound belonging to the class of quasi-binary $A^{I V} \mathrm{Te}_{-} \mathrm{Bi}_{2} \mathrm{Te}_{3}\left(A^{I V}=\mathrm{Pb}, \mathrm{Ge}\right.$, $\mathrm{Sn})$ series might be regarded as the intergrowths of $P b T e$ - type and $B i_{2} \mathrm{Te}_{3}$-type structures [16, 18-23]. The crystal structure of $\mathrm{PbBi}_{4} \mathrm{Te}_{7}$ is hexagonal with the primitive cell shown in Fig. 1 . We used the experimental crystal lattice constants [24,25], i.e. $a=4.46 \AA$ and $c=23.65 \AA$ for our calculations. To describe transport properties, we utilized the solution of the semi classical Boltzmann Transport Equation to estimate the transport coefficients [26] in which the electronic thermal conductivity was derived from the heat current. In that scene, it can be expressed in term of the integral of transport distribution function as following

$$
\kappa_{e}=\frac{1}{e^{2} T}\left(I T D_{i j}^{(2)}-\sum_{l, k=x, y, z} I T D_{i l}^{(1)} I T D_{l k}^{(0)-1} I T D_{k j}^{(1)}\right),
$$

where

$$
I T D_{i j}^{(\alpha)}=\int d \varepsilon \frac{\partial f}{\partial \varepsilon}(\varepsilon-\mu)^{\alpha} T D_{i j}(\varepsilon)
$$

is the integral of transport distribution function (ITD); TD is the transport distribution function defined by [26]

$$
T D_{i j}(\varepsilon)=e^{2} \sum_{\vec{k}} \tau_{i k}(\vec{k}) \delta(\varepsilon-\varepsilon(\vec{k})) \vec{v}_{k}(\vec{k}) \vec{v}_{j}(\vec{k})
$$

in which $\varepsilon$ is band energy, $e$ the elementary charge, $\tau$ the relaxation time constant, $\mu$ chemical potential, $f$ the Fermi-Dirac distribution function, $\delta$ Dirac delta function, $v$ group velocity. Such band dependent quantities, i.e. $\varepsilon$ and $v$, are derived from the ground state which were carried out from first-principle calculation within density functional theory. The dimensionless thermoelectric figure of merit can be therefore estimated by using Eq. (1). 


\section{RESULTS AND DISCUSSIONS}

$\mathrm{PbBi}_{4} \mathrm{Te}_{7}$ is a narrow band gap semiconductor. In our most precise calculation, as reported in Ref. [13], the band gap of the compound is of about $0.11 \mathrm{eV}$ obtained in the screened-exchange (sX) local-density approximation (LDA) with spin-orbital coupling (SOC) calculation (so called sX-LDA+SOC calculation). For the transport properties, we invoke the solution of the Boltzmann Transport Equation in which the eigenvalues are used to estimate the group velocity in Eq. (3). Accordingly, the eigenvalues which were conducted from a dense k-grid mesh in LDA+SOC calculation together with the use of the rigid band approximation [27] are used for further calculations. Thus, the bands above the Fermi energy are shifted to achieve the target band gap acquired in the sX-LDA+SOC calculation. To show, we illustrate in Fig. 2 such the band structure of $\mathrm{PbBi}_{4} \mathrm{Te}_{7}$ calculated by using LDA+SOC with the shifted sX-LDA+SOC band gap [13,28-30]. The p-type doped semiconductor is considered as the dominant effect of valence band on the desired property, e.g. the thermoelectric property. The dominant effect of conduction bands, i.e. n-type doping, will be considered in another work.

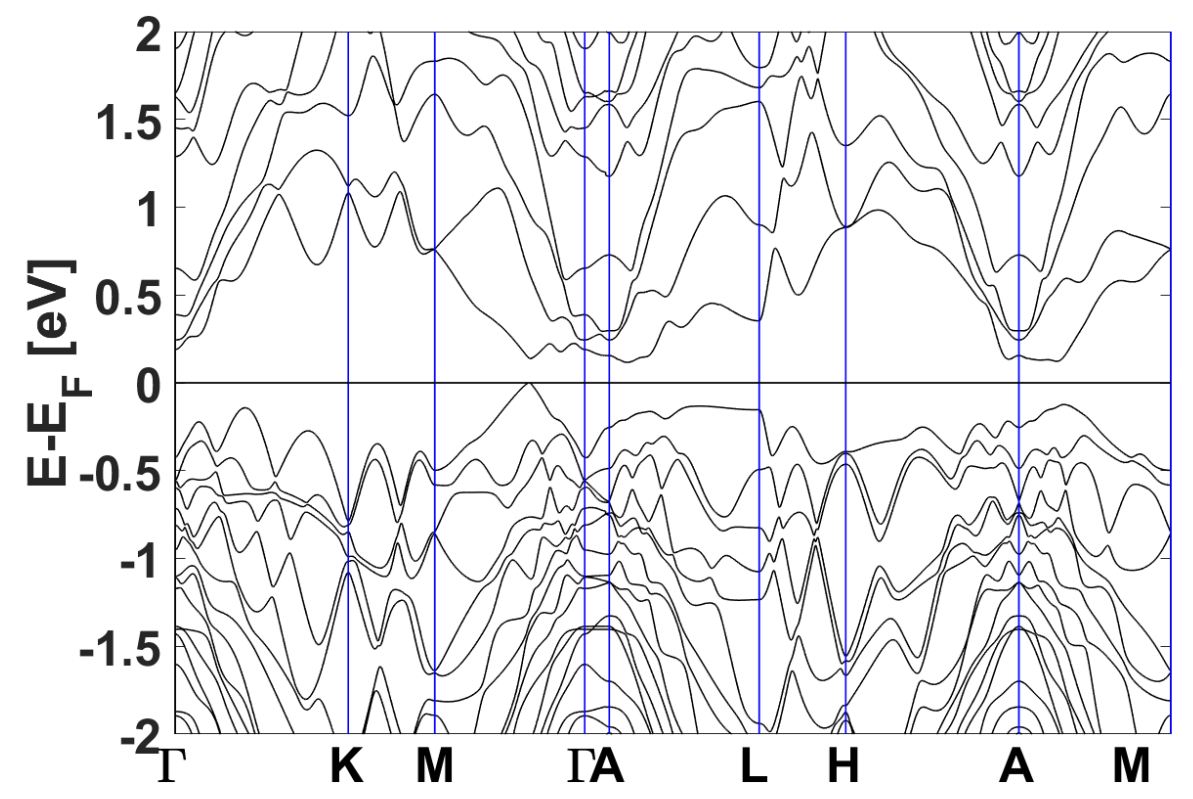

Fig. 2. Band structure of $\mathrm{PbBi}_{4} \mathrm{Te}_{7}$ calculated by using LDA-SOC with shifted band gap obtained from the self-consistent sX-LDA+SOC calculation.

The utmost important task appeared is to estimate the transport distribution function (see Eq. (3)). This task, as described, is carried out by calling the eigenvalues collected in the LDA+SOC calculation with the shifted SX-LDA+SOC band gap. The calculation of the transport coefficients, i.e. $\kappa_{e}, \sigma, \mathrm{S}$, etc. are therefore straight forward. In this report, we focus on $\kappa_{e}$ to analyze $Z T$ of $\mathrm{PbBi}_{4} \mathrm{Te}_{7}$. The electronic thermal conductivity (as well as the electrical conductivity) is proportional to the relaxation time constant, $\tau$, as shown in Eq. (2) and Eq. (3) within the relaxation 
time approximation. $\tau$ is an empirical parameter which is found by comparing some calculated quantities such as $S, \sigma$, or $Z T$ with available experimental quantities [10,30]. The typical $\tau$ for bismuth telluride and it alloys is about $10^{-14} s[10,14,28,30,31]$. It is noted that in the approximation concerned, $\kappa_{e} / \tau$ is relaxation time independence (see Eq. (2)). Accordingly, we present our results in term of $\kappa_{e} / \tau$ as a reduced electronic thermal conductivity. The results are presented in Fig. 3 which shows temperature (unit in K) and carrier concentration (unit in $\log _{10}, \mathrm{~cm}^{-3}$ ) dependences. The contour lines present $\kappa_{e}$ with the equal values. As clearly shown, $\kappa_{e}$ is drastically increased with the increase of the carrier concentration at a certain temperature. For a fixed carrier concentration, $\kappa_{e}$ is also gradually increased with temperature. It is noted that the power factor, $S^{2} \sigma$, has been reported to increase monotonically with the carrier concentration [13]. The increase of the power factor is likely to be linearly, the increase of $\kappa_{e}$ with a power-law become prominent to reduce $Z T$. This is a main reason for the constraint of $Z T$.

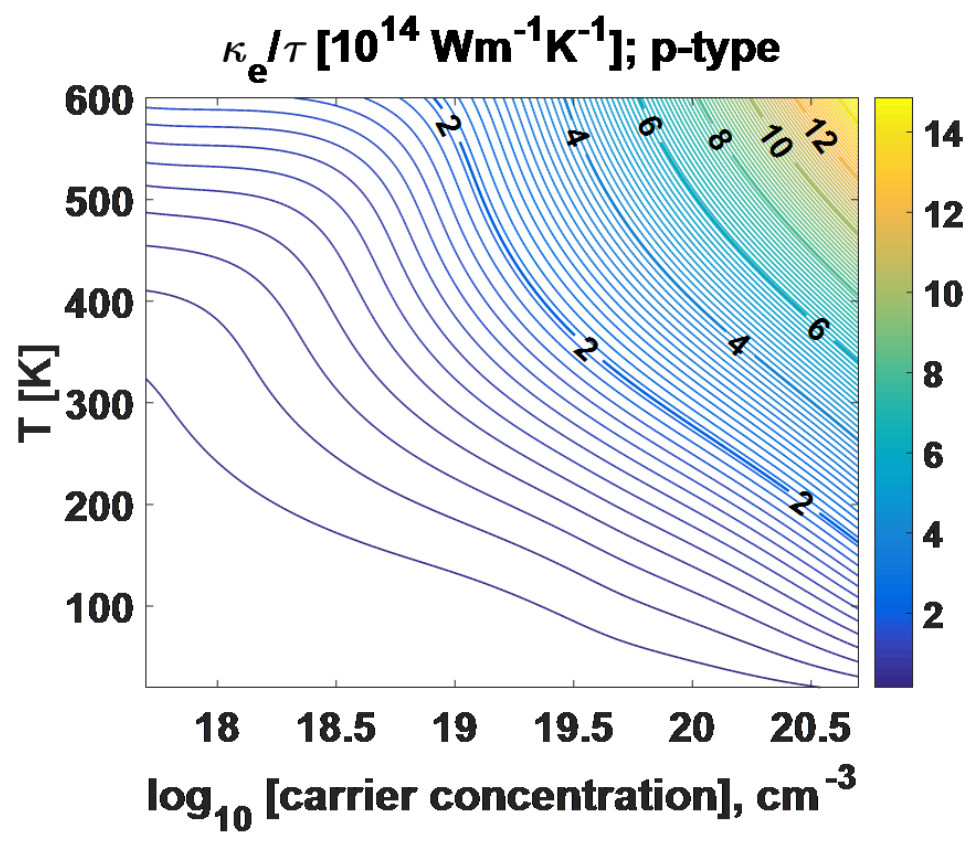

Fig. 3. (Color online) The calculated electronic thermal conductivity, $\kappa_{e} / \tau$, as a function of temperature (unit in $\mathrm{K}$ ) and carrier concentrations $\mathrm{n}$ (unit in $\log _{10}, \mathrm{~cm}^{-3}$ ).

Owing to the Wiedemann - Franz law [1,22], the increase of $\kappa$ leads to increase of $\sigma$. Thus, their opposite tendencies shape the thermoelectric efficiency, ZT. To substantiate this point, we compute $Z T$ as a two-variable function of temperature and carrier concentration. For this task, we assume that the compound is an ideal phonon-glass thermoelectric material of which the lattice thermal conductivity is much smaller than the electronic thermal conductivity, i.e. the ratio $\kappa_{L} / \kappa_{e}$ is negligible. Hence, the figure of merit approaches to the following value

$$
Z T=\frac{\sigma S^{2} T}{\kappa_{e}} .
$$




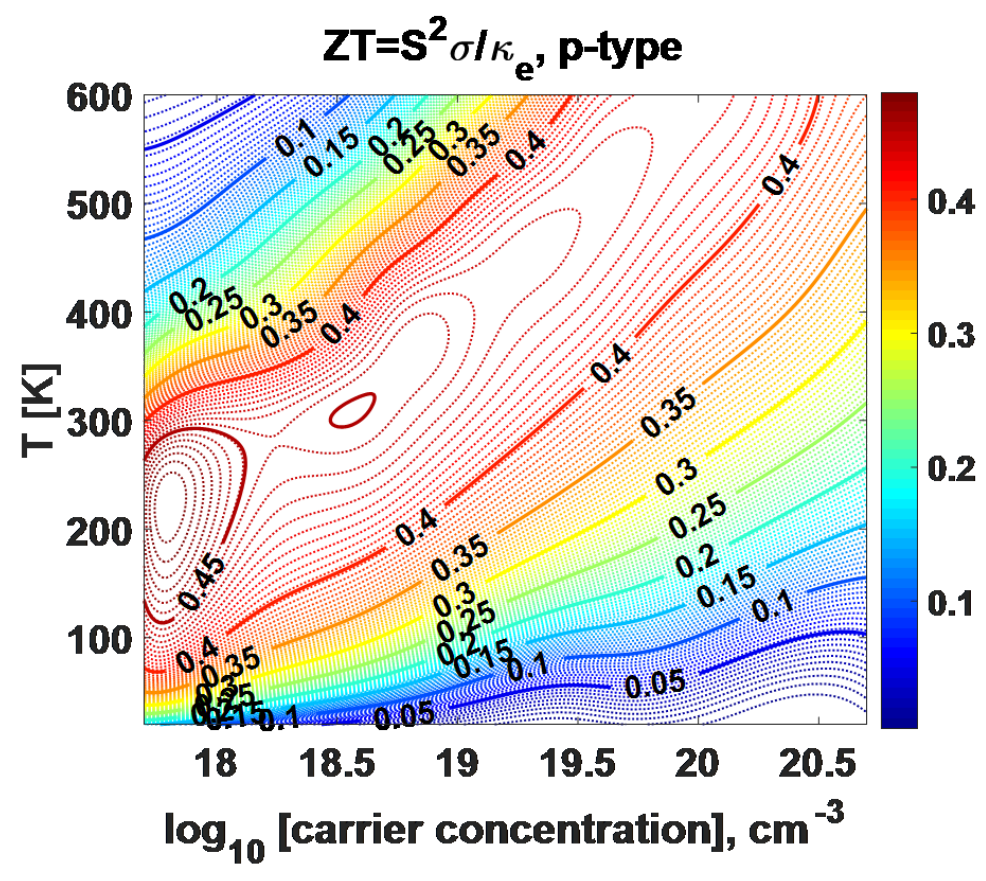

Fig. 4. (Color online) Temperature, $T$ (in $K$ ) and carrier concentration $n\left(\right.$ in $10^{19} \mathrm{~cm}^{-3}$ ) dependences of $Z T$.

It is noted that $\sigma / \kappa_{e}$ is relaxation time independence so is $Z T$. Fig. 4 presents our calculated results. As can be shown, there are two obvious peaks of ZT. One occurs at low carrier concentration and temperature, i.e. $6 \times 10^{17} \mathrm{~cm}^{-3}$ and $223 \mathrm{~K}$. This peak arises from the Seebeck coefficients [13]. At low temperatures and low carrier concentrations, the bipolar conduction is weak. Thus the band gap plays a great barrier to keep the intrinsic carriers from thermal excitation. This feature remains the Seebeck coefficient to be high enough [13]. In addition, $\kappa_{e}$ as shown above is very low in this area. The second peak occurs at higher carrier concentration and temperature, i.e. $3.6 \times 10^{18} \mathrm{~cm}^{-3}$ and $307 \mathrm{~K}$. This stems from the compromise between the gradual rise of $\sigma$ and $\kappa$ and the reduction of $S$. It also explains the reason why $Z T$ at this peak is slightly smaller than that at the former peak.

To elucidate the dependences, we explicitly calculate $Z T$ as a function of temperature at various carrier concentrations and as a function of carrier concentration at various temperatures. We present such these results in Fig. 5. As can be seen, at a fixed carrier concentration, $Z T$ almost increases monotonically with the temperature. In addition, Fig. 5a shows the monotonic decrease of $Z T$ with the increase of the carrier concentration. In contrast, Fig. $5 b$ shows that $Z T$ can be greatly improved with an optimized carrier concentration at a particular temperature. It is noted that at low temperatures, e.g. below $200 \mathrm{~K}$, the peak of $Z T$ in Fig. $5 \mathrm{~b}$ is sharp. It implies that $Z T$ is not stable with the change of the carrier concentration. In this fashion, the most stable $Z T$ is at around room temperature. At temperatures above room temperature, the peak of $Z T$ is gradually sharp. The peaks occurred at different temperatures show that the maximum value of $Z T$ may achieved with a relevant optimal carrier concentration. In Table 1, we list the optimal carrier 
concentration with various temperatures and the corresponding maximum $Z T$ values. As shown, the highest maximum $Z T$ of 0.48 is reached at a low temperature, i.e. $220 \mathrm{~K}$ and at the optimal carrier concentration of $6.0 \times 10^{17} \mathrm{~cm}^{-3}$. At room temperature, the optimized $Z T$ slightly reduces to be 0.47 . And at temperatures around $400 \mathrm{~K}, Z T$ is of about 0.44 . This result is in accordance with experimental observation in which $Z T$ was reported to be $\sim 0.4$ at $500 \mathrm{~K}$ [15]. We also note that our maximum $Z T$ achieved by optimizing carrier concentration is significantly improved in comparison with experimental $Z T$ of 0.01 at $300 \mathrm{~K}$ [17]. This indicates that at a particular temperature, to improve $Z T$ the carrier concentration must be carefully optimized, especially at low carrier concentrations, e.g. around $6.0 \times 10^{17} \mathrm{~cm}^{-3}$. By taking into account the effect of electronic thermal conductivity, we suggest that $\mathrm{PbBi}_{4} \mathrm{Te}_{7}$ with p-type doping is a promising TE candidate for thermoelectric application operating at around room temperature. Careful analyses and calculations finding out the effect of impurities, defects, etc. which yield p-type doping on ZT were not considered and appeared to be an interesting issue for future studies.

Table 1. The maximum $Z T$ at various temperatures $\mathrm{T}$ (unit in $\mathrm{K}$ ) at appropriate optimized carrier concentration $\mathrm{n}_{\text {opt }}$ (unit in $10^{19} \mathrm{~cm}^{-3}$ ).

\begin{tabular}{lrrrrrr}
\hline$T(\mathrm{~K})$ & 100 & 200 & 223 & 300 & 400 & 500 \\
\hline$n_{\text {opt }}\left(10^{19} \mathrm{~cm}^{-3}\right)$ & 0.05 & 0.06 & 0.06 & 0.20 & 0.87 & 3.36 \\
\hline$Z T$ & 0.43 & 0.47 & 0.48 & 0.47 & 0.44 & 0.44 \\
\hline
\end{tabular}

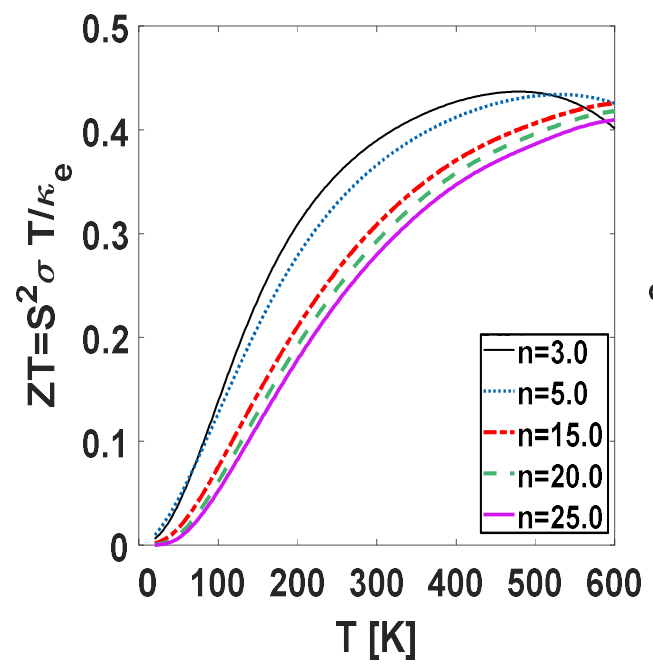

(a)

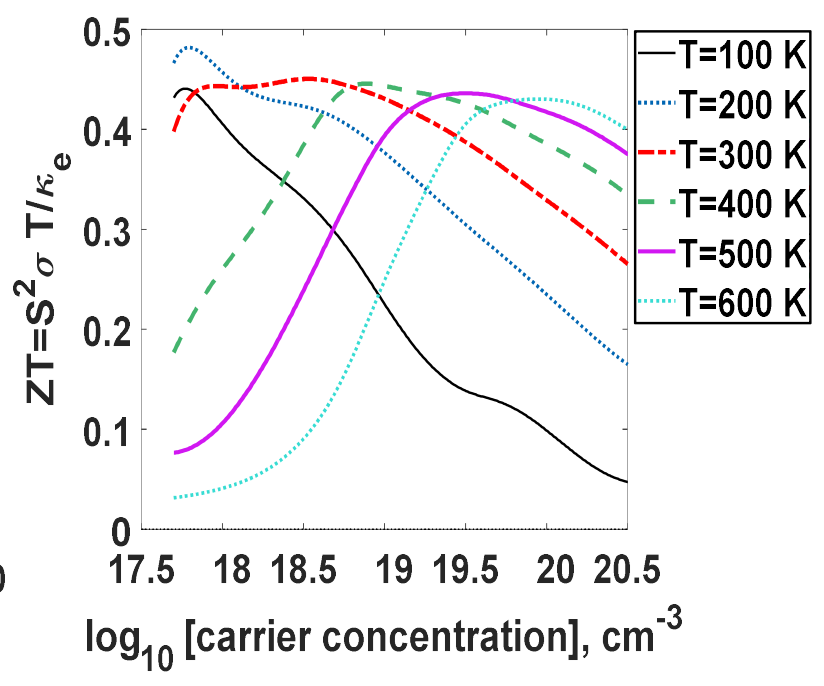

(b)

Fig. 5. (Color online) Calculation of $Z T=S^{2} \sigma T / \kappa_{e}$ as a function of (a) temperature at various carrier concentrations $\mathrm{n}$ (unit in $10^{19} \mathrm{~cm}^{-3}$ ) and (b) carrier concentration (unit in $\left.\log _{10}, \mathrm{~cm}^{-3}\right)$ at various temperatures. 


\section{CONCLUSIONS}

We reported the effect of the electronic thermal conductivity on thermoelectric properties of the quasi-binary compound, $\mathrm{PbBi}_{4} \mathrm{Te}_{7}$, with p-type doping by employing the solution of the Boltzmann Transport Equation in a constant relaxation-time approximation. We found that the increase of carrier concentration leads to a drastic increase of the electronic thermal conductivity. Therefore, it lowers the thermoelectric performance. Nevertheless, at a fixed temperature, ZT can be improved significantly by optimizing carrier concentration. Assuming the ideal phonon-glass, we computed $Z T$ and obtained that the maximum $Z T$ can reach 0.48 at a low temperature, i.e. 220 $\mathrm{K}$ and at carrier concentration of $6.0 \times 10^{17} \mathrm{~cm}^{-3}$. At room temperature, the optimized $Z T$ slightly reduces with the value of around 0.47. Our findings suggest that $\mathrm{PbBi}_{4} \mathrm{Te}_{7}$ with p-type doping is a promising TE materials operating at around room temperature.

\section{ACKNOWLEDGMENTS}

This research is funded by Vietnam National Foundation for Science and Technology Development (NAFOSTED) under grant number 103.01-2015.11. We also would like to thank Professor Miyoung Kim at Sookmyung's Women University for her advice.

\section{REFERENCES}

[1] G. Chen, M.S. Dresselhaus, G. Dresselhaus, J.-P. Fleurial, T. Caillat, Int. Mater. Rev. 48 (2013) 45.

[2] T. Takabatake, K. Suekuni, T. Nakayama, E. Kaneshita, Rev. Mod. Phys. 86 (2014) 669.

[3] X. Zhang, L.-D. Zhao, J. Mater. 1 (2015) 92.

[4] G. J. Snyder, E. S. Toberer, Nat. Mater. 7 (2008) 105.

[5] R. Venkatasubramanian, E. Siivola, T. Colpitts, B. O’Quinn, Nature 413 (2001) 597.

[6] T. Van Quang, M. Kim, J. Appl. Phys. 120 (2016) 195105.

[7] A. Bulusu, D.G. Walker, Superlattices Microstruct. 44 (2008) 1.

[8] C. Gayner and K. K. Kar, Prog. Mater. Sci. 83 (2016) 330.

[9] T. Van Quang and M. Kim, J. Appl. Phys. 113 (2013) 17A934.

[10] T. Van Quang and M. Kim, IEEE Trans. Magn. 50 (2014) 1000904.

[11] K. Hoang, S.D. Mahanti, M.G. Kanatzidis, Phys. Rev. B 81 (2010) 115106.

[12] M. K. Zhitinskaya, S. A. Nemov, A. A. Muhtarova, L. E. Shelimova, T. E. Svechnikova and P. P. Konstantinov, Semiconductors 44 (2010) 729.

[13] T. Van Quang, K. Miyoung, J. Korean Phys. Soc. 68 (2016) 393.

[14] L. Zhang, D. J. Singh, Phys. Rev. B 81 (2010) 245119.

[15] V. L. Kuznetsov, L. A. Kuznetsova and D.M. Rowe, J. Phys. D. Appl. Phys. 34 (2001) 700.

[16] L. E. Shelimova, T. E. Svechnikova, P. P. Konstantinov, O. G. Karpinskii, E. S. Avilov, M. a. Kretova and V.S. Zemskov, Inorg. Mater. 43 (2007) 125.

[17] M. K. Zhitinskaya, S. A. Nemov, N. M. Blagih, L. E. Shelimova, T. E. Svechnikova, Semiconductors 46 (2012) 1256.

[18] L. E. Shelimova, O.G. Karpinskii, P. P. Konstantinov, E. S. Avilov, M. A. Kretova and V. S. Zemskov, Inorg. Mater. 40 (2004) 451.

[19] L. E. Shelimova, O. G. Karpinskii, P. P. Konstantinov, M. A. Kretova, E. S. Avilov and V. S. Zemskov, Inorg. Mater. 38 (2002) 790.

[20] V. S. Zemskov, L. E. Shelimova, P. P. Konstantinov, E. S. Avilov, M. a. Kretova and I. Y. Nikhezina, Inorg. Mater. Appl. Res. 3 (2012) 61.

[21] P. P. Konstantinov, L. E. Shelimova, E. S. Avilov, M. A. Kretova and J.-P. Fleurial, J. Solid State Chem. 146 (1999) 305.

[22] F. N. Guseinov, M. B. Babanly, V. P. Zlomanov and Y. A. Yusibov, Russ. J. Inorg. Chem. 57 (2012) 1387. 
[23] M. B. Babanly, A. V. Shevel'kov, F. N. Guseinov, and D. M. Babanly, Inorg. Mater. 47 (2011) 712.

[24] I. I. Petrov, R.M. Imamov, Sov. Phys. Crystallogr. 14 (1969) 699.

[25] Y. Imai and A. Watanabe, Intermetallics 11 (2003) 451.

[26] G. D. Mahan and J.O. Sofo, Proc. Natl. Acad. Sci. U. S. A. 93 (1996) 7436.

[27] J. L. P. Hughes and J. E. Sipe, Phys. Rev. B 53 (1996) 10751.

[28] M. S. Park, J. H. Song, J. E. Medvedeva, M. Kim, I. G. Kim and A. J. Freeman, Phys. Rev. B 81 (2010) 155211.

[29] T. Van Quang, M. Kim, J. Appl. Phys. 122 (2017) 245104.

[30] T. Quang, H. Lim and M. Kim, J. Korean Phys. Soc. 61 (2012) 1728.

[31] T. Thonhauser and T. J. Scheidemantel, J. O. Sofo, Appl. Phys. Lett. 85 (2004) 588.

[32] G. V. Chester, A. Thellung, Proc. Phys. Soc. 77 (1961) 1005. 